\title{
The importance of cylinder passivation for preparation and long-term stability of multicomponent monoterpene primary reference materials
}

Nicholas D. C. Allen et al.

Correspondence to: Nicholas D. C. Allen (nick.allen@npl.co.uk)

The copyright of individual parts of the supplement might differ from the CC BY 4.0 License. 


\section{Description of uncertainty analysis procedure}

Below is the uncertainty evaluation adopted when comparing the response of an unknown mixture against a validated calibration standard e.g. a PRM:

$$
\overline{\mathrm{r}}=\frac{2 \mathrm{~A}_{\mathrm{u}, \mathrm{avg}}}{\left(\mathrm{A}_{\mathrm{s}, \mathrm{avg} 1}+\mathrm{A}_{\mathrm{s}, \mathrm{avg} 2}\right)}
$$

Where $\overline{\mathrm{r}}$ is the average ratio, $A_{u, \text { avg }}$ is the average peak area from $\mathrm{n}$ repeated measurements of the comparison mixture, $A_{s, a v g l}$ is the average peak area from $\mathrm{n}$ repeated measurements of the calibration standard before running the comparison mixture and $A_{s, a v g 2}$ is the average peak area from $\mathrm{n}$ repeated measurements of the calibration standard after running the comparison mixture.

The amount fraction of the target component in the comparison mixture, $x_{u}$, is then calculated by:

$$
\mathrm{x}_{\mathrm{u}}=\mathrm{x}_{\mathrm{s}} \overline{\mathrm{r}}
$$

Where $x_{s}$ is the amount fraction of the target component in the standard. The standard uncertainty of the measurand, $u\left(x_{u}\right)$, is calculated by:

$$
\frac{\mathrm{u}\left(\mathrm{x}_{\mathrm{u}}\right)}{\mathrm{x}_{\mathrm{u}}}=\sqrt{\frac{\mathrm{u}\left(\mathrm{x}_{\mathrm{s}}\right)^{2}}{\mathrm{x}_{\mathrm{s}}{ }^{2}}+\frac{\mathrm{u}(\overline{\mathrm{r}})^{2}}{\mathrm{r}^{2}}}
$$

$\mathrm{u}\left(x_{S}\right)$ is the uncertainty of the reference standard $\mathrm{u}(\bar{r})$ is the uncertainty of the ratio, it includes e.g. repeatability, internal blanks, peak shape (error of integration), error in the sample volume. The uncertainty in $\bar{r}$ is calculated by:

$$
\frac{\mathrm{u}(\overline{\mathrm{r}})^{2}}{\overline{\mathrm{r}}^{2}}=\frac{\mathrm{u}\left(\mathrm{A}_{\mathrm{u}, \mathrm{avg}}\right)^{2}}{\mathrm{~A}_{\mathrm{u}, \mathrm{avg}}{ }^{2}}+\frac{\mathrm{u}\left(\mathrm{A}_{\mathrm{s}, \mathrm{avg} 1}\right)^{2}}{\left(\mathrm{~A}_{\mathrm{s}, \mathrm{avg} 1}+A_{\mathrm{s}, \mathrm{avg} 2}\right)^{2}}+\frac{\mathrm{u}\left(\mathrm{A}_{\mathrm{s}, \mathrm{avg} 2}\right)^{2}}{\left(\mathrm{~A}_{\mathrm{s}, \mathrm{avg} 1}+\mathrm{A}_{\mathrm{s}, \mathrm{avg} 2}\right)^{2}}
$$


Table S1: Overview of purity analysis of chemicals purchased.

\begin{tabular}{|c|c|c|c|c|c|c|c|c|}
\hline & \multicolumn{8}{|c|}{ Chemical purity analysed } \\
\hline $\begin{array}{l}\text { Compounds } \\
\text { found }\end{array}$ & $\begin{array}{l}+-\alpha- \\
\text { pinene }\end{array}$ & $\begin{array}{l}--\alpha- \\
\text { pinene }\end{array}$ & $\begin{array}{l}\text { limone } \\
\text { ne }\end{array}$ & $\begin{array}{l}\text { 3- } \\
\text { carene }\end{array}$ & $\begin{array}{l}\text { 1,8- } \\
\text { cineole }\end{array}$ & $\begin{array}{l}n- \\
\text { octane }\end{array}$ & $\begin{array}{l}+-\beta- \\
\text { pinene }\end{array}$ & $\begin{array}{l}--\beta- \\
\text { pinene }\end{array}$ \\
\hline$\pm \alpha$-pinene & $97.95 \%$ & $98.80 \%$ & $<0.01 \%$ & $0.02 \%$ & $0.03 \%$ & $<0.01 \%$ & $1.61 \%$ & $1.08 \%$ \\
\hline$\pm \beta$-pinene & $0.06 \%$ & $0.11 \%$ & $<0.01 \%$ & $0.02 \%$ & $0.03 \%$ & $<0.01 \%$ & $93.82 \%$ & $95.33 \%$ \\
\hline limonene & $0.17 \%$ & $0.24 \%$ & $99.04 \%$ & $0.24 \%$ & $0.34 \%$ & $<0.01 \%$ & $1.48 \%$ & $0.87 \%$ \\
\hline 3-carene & $<0.01 \%$ & $<0.01 \%$ & $<0.01 \%$ & $98.23 \%$ & $<0.01 \%$ & $<0.01 \%$ & $<0.01 \%$ & $<0.01 \%$ \\
\hline 1,8-cineole & $<0.01 \%$ & $<0.01 \%$ & $<0.01 \%$ & $<0.01 \%$ & $99.49 \%$ & $<0.01 \%$ & $<0.01 \%$ & $<0.01 \%$ \\
\hline 3-carene & $<0.01 \%$ & $<0.01 \%$ & $<0.01 \%$ & $<0.01 \%$ & $0.01 \%$ & $<0.01 \%$ & $<0.01 \%$ & $<0.01 \%$ \\
\hline cis-ocimene & $0.01 \%$ & $0.03 \%$ & $0.02 \%$ & $<0.01 \%$ & $<0.01 \%$ & $<0.01 \%$ & $<0.01 \%$ & $<0.01 \%$ \\
\hline myrcene & $<0.01 \%$ & $<0.01 \%$ & $0.01 \%$ & $<0.01 \%$ & $<0.01 \%$ & $<0.01 \%$ & $<0.01 \%$ & $<0.01 \%$ \\
\hline$n$-octane & $<0.01 \%$ & $<0.01 \%$ & $<0.01 \%$ & $<0.01 \%$ & $<0.01 \%$ & $99.90 \%$ & $<0.01 \%$ & $<0.01 \%$ \\
\hline $\begin{array}{l}\text { unknown } \\
\text { terpene }\end{array}$ & $0.60 \%$ & $0.42 \%$ & $0.30 \%$ & $0.96 \%$ & $0.04 \%$ & $<0.01 \%$ & $3.07 \%$ & $2.71 \%$ \\
\hline $\begin{array}{l}\text { other } \\
\text { hydrocarbons }\end{array}$ & $1.21 \%$ & $0.27 \%$ & $0.63 \%$ & $0.53 \%$ & $0.06 \%$ & $0.10 \%$ & $0.02 \%$ & $0.03 \%$ \\
\hline
\end{tabular}

Table S2: The percentage difference between the reference standard mixture BB and the decanted monoterpene mixture in a $10 \mathrm{~L}$ internally passivated Experis cylinder (normalised for gravimetric differences).

\begin{tabular}{|l|l|c|c|c|c|c|}
\hline \multicolumn{2}{|c|}{ Experis } & \multicolumn{3}{c|}{ Difference with respect to the reference standard mixture BB } \\
\hline Decant & Pressure & n-octane & $\begin{array}{l}\boldsymbol{+}-\boldsymbol{\alpha -} \\
\text { pinene }\end{array}$ & 3-carene & R-limonene & $\begin{array}{l}\text { 1,8- } \\
\text { cineole }\end{array}$ \\
\hline cylinder 1 & $120 \mathrm{bar}$ & $0.0 \%$ & $0.0 \%$ & $0.6 \%$ & $0.7 \%$ & $0.5 \%$ \\
\hline cylinder 1 & $70 \mathrm{bar}$ & $0.7 \%$ & $0.4 \%$ & $0.3 \%$ & $0.9 \%$ & $0.9 \%$ \\
\hline cylinder 2 & $50 \mathrm{bar}$ & $0.7 \%$ & $0.3 \%$ & $0.2 \%$ & $0.9 \%$ & $0.8 \%$ \\
\hline cylinder 2 & $30 \mathrm{bar}$ & $0.6 \%$ & $0.0 \%$ & $0.3 \%$ & $0.9 \%$ & $1.7 \%$ \\
\hline cylinder 3 & $20 \mathrm{bar}$ & $0.3 \%$ & $-0.8 \%$ & $-0.6 \%$ & $-0.4 \%$ & $-1.1 \%$ \\
\hline
\end{tabular}

Table S3: The percentage difference between the reference standard mixture BB and the decanted monoterpene mixture in a $10 \mathrm{~L}$ internally passivated Experis cylinder repeated (normalised for gravimetric differences).

\begin{tabular}{|l|l|c|c|c|c|c|}
\hline \multicolumn{2}{|c|}{ Experis repeat } & \multicolumn{3}{c|}{ Difference with respect to the reference standard mixture BB } \\
\hline Decant & Pressure & n-octane & $\begin{array}{l}\boldsymbol{+} / \mathbf{\alpha -} \\
\text { pinene }\end{array}$ & 3-carene & R-limonene & $\begin{array}{l}\mathbf{1 , 8} \text { - } \\
\text { cineole }\end{array}$ \\
\hline cylinder 1 & $120 \mathrm{bar}$ & $0.5 \%$ & $0.7 \%$ & $0.9 \%$ & $1.4 \%$ & $1.5 \%$ \\
\hline cylinder 1 & $70 \mathrm{bar}$ & $0.5 \%$ & $0.8 \%$ & $0.8 \%$ & $0.6 \%$ & $0.8 \%$ \\
\hline cylinder 2 & $50 \mathrm{bar}$ & $0.3 \%$ & $0.6 \%$ & $0.5 \%$ & $0.9 \%$ & $1.0 \%$ \\
\hline cylinder 2 & $30 \mathrm{bar}$ & $0.1 \%$ & $-0.1 \%$ & $0.0 \%$ & $-0.2 \%$ & $-0.1 \%$ \\
\hline cylinder 3 & $20 \mathrm{bar}$ & $0.6 \%$ & $0.4 \%$ & $0.3 \%$ & $0.5 \%$ & $-0.2 \%$ \\
\hline
\end{tabular}


Table S4: The percentage difference between the reference standard mixture BB and the decanted monoterpene mixture in a $10 \mathrm{~L}$ internally passivated BOC SPECTRASEAL cylinder (normalised for gravimetric differences).

\begin{tabular}{|c|c|c|c|c|c|c|}
\hline \multicolumn{2}{|c|}{ BOC SPECTRA-SEAL } & \multicolumn{5}{|c|}{ Difference with respect to the reference standard mixture BB } \\
\hline Decant & Pressure & $n$-octane & $\begin{array}{l}+/-\alpha- \\
\text { pinene }\end{array}$ & +3 -carene & R-limonene & $\begin{array}{l}\text { 1,8- } \\
\text { cineole }\end{array}$ \\
\hline cylinder 1 & 120 bar & $0.3 \%$ & $-61.8 \%$ & $-2.0 \%$ & $8.5 \%$ & $-20.4 \%$ \\
\hline cylinder 1 & 70 bar & \multirow{4}{*}{\multicolumn{5}{|c|}{$\begin{array}{l}\text { No further decants were performed for this cylinder type as the } \\
\text { passivation was shown to be unsuitable for monoterpenes. }\end{array}$}} \\
\hline cylinder 2 & 50 bar & & & & & \\
\hline cylinder 2 & 30 bar & & & & & \\
\hline cylinder 3 & 20 bar & & & & & \\
\hline
\end{tabular}

Table S5: The percentage difference between the reference standard mixture BB and the decanted monoterpene mixture in a $10 \mathrm{~L}$ internally passivated BOC SPECTRASEAL cylinder (normalised for gravimetric differences) repeated.

\begin{tabular}{|c|c|c|c|c|c|c|}
\hline \multicolumn{2}{|c|}{$\begin{array}{c}\text { BOC SPECTRA-SEAL } \\
\text { repeat }\end{array}$} & \multicolumn{5}{|c|}{ Difference with respect to the reference standard mixture BB } \\
\hline Decant & Pressure & $n$-octane & $\begin{array}{l}+/-\alpha- \\
\text { pinene }\end{array}$ & +3-carene & R-limonene & $\begin{array}{l}1,8- \\
\text { cineole }\end{array}$ \\
\hline cylinder 1 & 120 bar & $0.4 \%$ & $-100.0 \%$ & $-26.5 \%$ & $-53.5 \%$ & $-52.0 \%$ \\
\hline cylinder 1 & 70 bar & \multirow{4}{*}{\multicolumn{5}{|c|}{$\begin{array}{l}\text { No further decants were performed for this cylinder type as the } \\
\text { passivation was shown to be unsuitable for monoterpenes. }\end{array}$}} \\
\hline cylinder 2 & 50 bar & & & & & \\
\hline cylinder 2 & 30 bar & & & & & \\
\hline cylinder 3 & 20 bar & & & & & \\
\hline
\end{tabular}

Table S6: The percentage difference between the reference standard mixture BB and the decanted monoterpene mixture in a $10 \mathrm{~L}$ internally passivated treated BOC SPECTRA-SEAL cylinder with further proprietary in-house treatment (normalised for gravimetric differences).

\begin{tabular}{|c|c|c|c|c|c|c|}
\hline \multicolumn{2}{|c|}{$\begin{array}{l}\text { In-house treated BOC } \\
\text { SPECTRA-SEAL }\end{array}$} & \multicolumn{5}{|c|}{ Difference with respect to the reference standard mixture BB } \\
\hline Decant & Pressure & $n$-octane & $\begin{array}{l}+/-\alpha- \\
\text { pinene }\end{array}$ & +3-carene & R-limonene & $\begin{array}{l}1,8- \\
\text { cineole }\end{array}$ \\
\hline cylinder 1 & 120 bar & $0.7 \%$ & $-100.0 \%$ & $-27.8 \%$ & $-48.8 \%$ & $-54.9 \%$ \\
\hline cylinder 1 & 70 bar & \multirow{4}{*}{\multicolumn{5}{|c|}{$\begin{array}{l}\text { No further decants were performed for this cylinder type as the } \\
\text { passivation was shown to be unsuitable for monoterpenes. }\end{array}$}} \\
\hline cylinder 2 & 50 bar & & & & & \\
\hline cylinder 2 & 30 bar & & & & & \\
\hline cylinder 3 & 20 bar & & & & & \\
\hline
\end{tabular}


Table S7: The percentage difference between the reference standard mixture BB and the decanted monoterpene mixture in a $10 \mathrm{~L}$ internally passivated treated BOC SPECTRA-SEAL cylinder with further proprietary in-house treatment (normalised for gravimetric differences) repeated.

\begin{tabular}{|c|c|c|c|c|c|c|}
\hline \multicolumn{2}{|c|}{$\begin{array}{l}\text { In-house treated BOC } \\
\text { SPECTRA-SEAL repeat }\end{array}$} & \multicolumn{5}{|c|}{ Difference with respect to the reference standard mixture BB } \\
\hline Decant & Pressure & $n$-octane & $\begin{array}{l}+/-\alpha- \\
\text { pinene }\end{array}$ & +3-carene & R-limonene & $\begin{array}{l}\text { 1,8- } \\
\text { cineole }\end{array}$ \\
\hline cylinder 1 & 120 bar & $0.1 \%$ & $-100.0 \%$ & $-94.9 \%$ & $-94.5 \%$ & $-97.4 \%$ \\
\hline cylinder 1 & 70 bar & \multirow{4}{*}{\multicolumn{5}{|c|}{$\begin{array}{l}\text { No further decants were performed for this cylinder type as the } \\
\text { passivation was shown to be unsuitable for monoterpenes. }\end{array}$}} \\
\hline cylinder 2 & 50 bar & & & & & \\
\hline cylinder 2 & 30 bar & & & & & \\
\hline cylinder 3 & 20 bar & & & & & \\
\hline
\end{tabular}

Table S8: Elution times, forward match (FM) and reverse match (RM) values obtained by mass spectrometry for Mixture BB.

\begin{tabular}{|l|c|c|c|}
\hline \multicolumn{4}{|c|}{ Mixture BB } \\
\hline Compound & Elution time & Forward match & Reverse match \\
\hline $\boldsymbol{\alpha}$-pinene & 39.25 & 904 & 907 \\
\hline 3-carene & 41.25 & 909 & 952 \\
\hline limonene & 41.65 & 894 & 896 \\
\hline 1,8-cineole & 41.80 & 864 & 900 \\
\hline
\end{tabular}

Table S9: Elution times, forward match (FM) and reverse match (RM) values obtained by mass spectrometry for a terpene mixture in a BOC SPECTRA-SEAL passivated cylinder.

\begin{tabular}{|l|c|c|c|}
\hline \multicolumn{4}{|c|}{ Mixture in BOC SPECTRA-SEAL passivated cylinder } \\
\hline Compound & Elution time & Forward match & Reverse match \\
\hline camphene & 39.80 & 946 & 962 \\
\hline $\boldsymbol{\alpha}$-terpinene & 41.40 & 921 & 930 \\
\hline cymene & 41.65 & 937 & 949 \\
\hline $\boldsymbol{\tau}$-terpinene & 42.25 & 911 & 931 \\
\hline terpinolene & 43.05 & 938 & 945 \\
\hline
\end{tabular}


Table S10: The Kovats' Retention Indices on a non-polar column, using a custom temperature program for reference publications 1-8 (Adams, 1998; Araujo et al., 2003; de Marchese et al., 2007; Dwivedi et al., 2004; Frizzo et al., 2001; Novak et al., 2001; Riu-Aumatell et al., 2004; Tuberoso et al., 2005).

\begin{tabular}{|c|c|c|c|c|c|c|c|c|c|c|c|c|}
\hline & & \multicolumn{11}{|c|}{ Kovats' Retention Indicies value } \\
\hline Elution time & Compound & 1 & 2 & 3 & 4 & 5 & 6 & 7 & 8 & Average & Max value & Min value \\
\hline 39.25 & $\alpha$-pinene & 938 & 937 & 933 & & 928 & 934 & 938 & 939 & 935 & 939 & 928 \\
\hline 39.80 & camphene & 954 & & 946 & & 955 & & 954 & 953 & 952 & 955 & 946 \\
\hline 41.25 & 3-carene & & & 1011 & & & 1011 & 1013 & 1011 & 1012 & 1013 & 1011 \\
\hline 41.40 & $\alpha$-terpinene & & 1017 & & & 1024 & & 1020 & 1018 & 1020 & 1024 & 1017 \\
\hline 41.65 & p-cymene & 1025 & 1026 & & & 1015 & 1023 & & 1023 & 1022 & 1026 & 1015 \\
\hline 41.66 & limonene & 1029 & 1029 & 1020 & 1017 & 1028 & 1029 & 1034 & 1031 & 1027 & 1034 & 1017 \\
\hline 41.80 & 1,8-cineole & & 1033 & 1020 & & & 1036 & 1031 & & 1030 & 1036 & 1020 \\
\hline 42.25 & $\gamma$-terpinene & & 1058 & 1051 & 1057 & 1058 & 1059 & 1063 & 1062 & 1058 & 1063 & 1051 \\
\hline 43.05 & terpinolene & & 1084 & 1097 & 1074 & 1086 & 1088 & 1092 & 1088 & 1087 & 1097 & 1074 \\
\hline
\end{tabular}



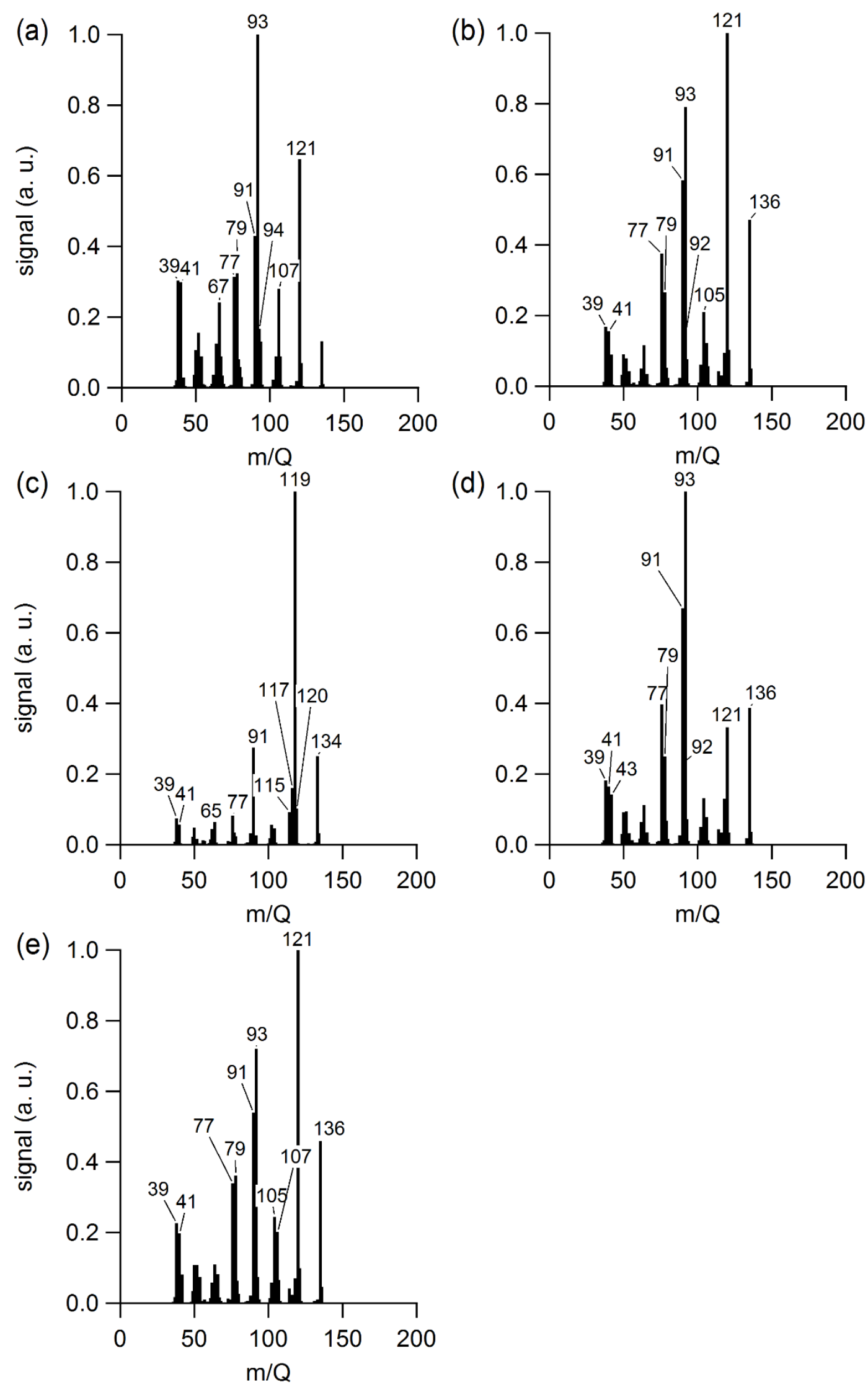

Figure S1: Mass spectrometry ion fragmentation spectra for (a) camphene (b) $\alpha$-terpinene (c) cymene (d) $\tau$-terpinene (e) terpinolene peaks identified and observed in a BOC SPECTRASEAL passivated cylinder. 


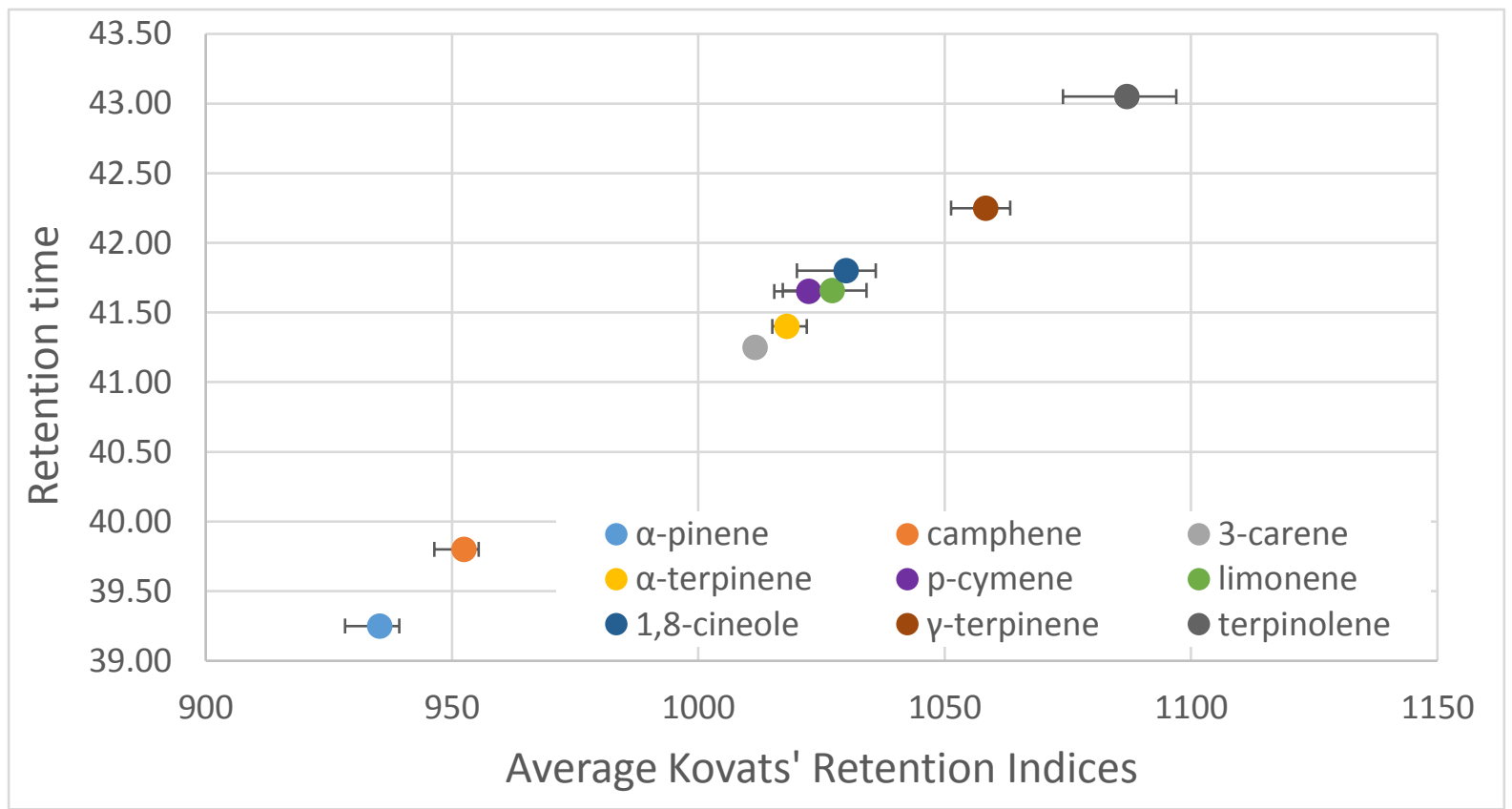

Figure S2: The average Kovats' Retention Indices on a non-polar column, using a custom temperature program for reference publications 1-8 (Adams, 1998; Araujo et al., 2003; de Marchese et al., 2007; Dwivedi et al., 2004; Frizzo et al., 2001; Novak et al., 2001; RiuAumatell et al., 2004; Tuberoso et al., 2005). Error bars cover the range from minimum to maximum for the Kovats' Retention Indice values.

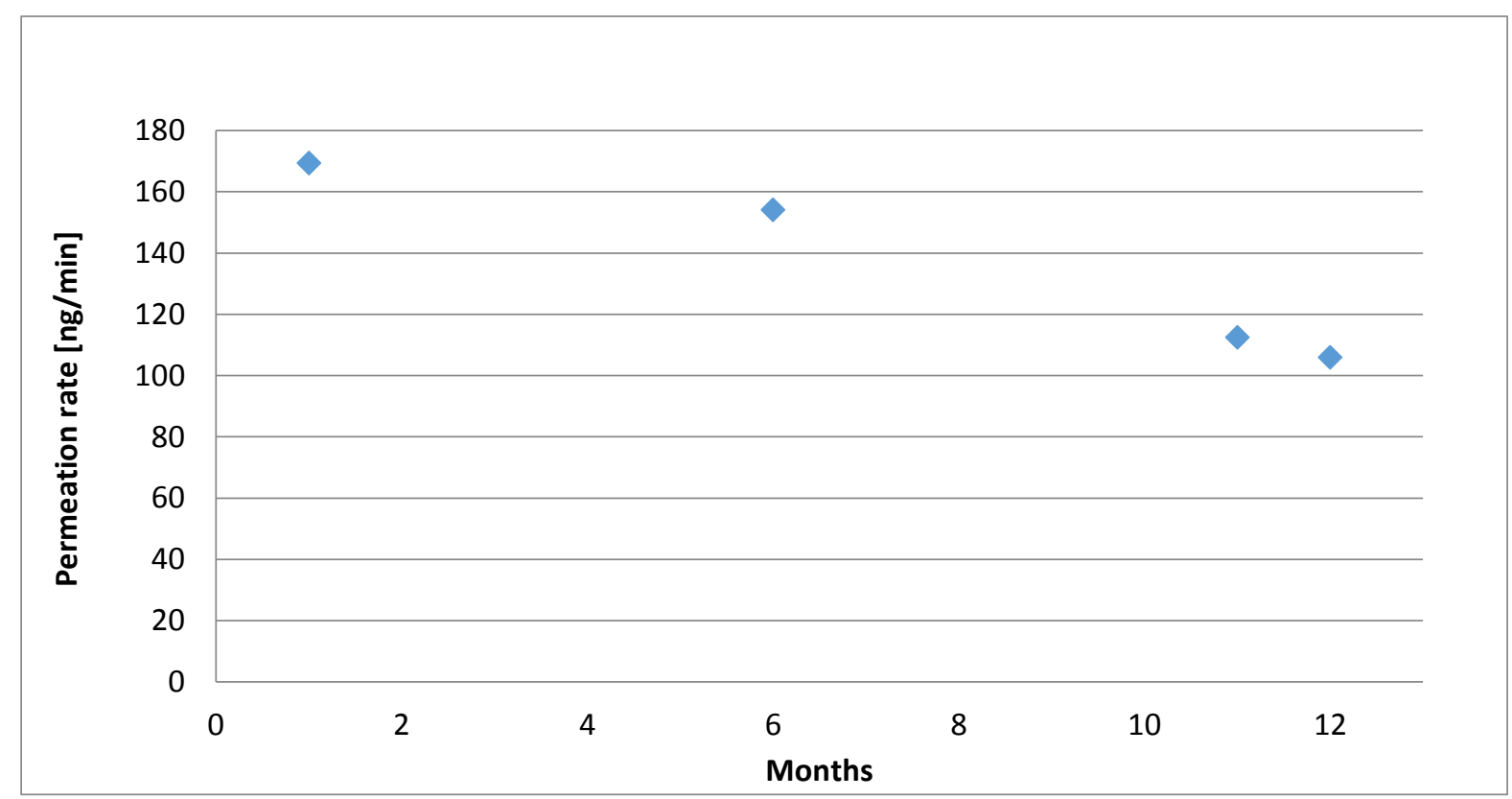

Figure S3: The permeation rate of limonene measured in the magnetic suspension balance over an 11 month period at $30^{\circ} \mathrm{C}$ with a nitrogen flow of $0.17 \mathrm{l} / \mathrm{min}$. 
Adams, R. P.: The leaf essential oils and chemotaxonomy of Juniperus sect. Juniperus, Biochem. Syst. Ecol., 26, 637-645, 1998.

Araujo, E. C. C., Silveira, E. R., Lima, M. A. S., Neto, M. A., de Andrade, I. L., Lima, M. A. A., Santiago, G. M. P., and Mesquita, A. L. M.: Insecticidal activity and chemical composition of volatile oils from Hyptis martiusii Benth, J. Agric. Food Chem., 51, 3760-3762, 2003.

de Marchese, M. J. A., de Heluani, C. S., Catalan, C. A. N., Griffin, C. A., Vaughn, J. B., and Herz, W.: Incisol, an alcohol with a novel sesquiterpene skeleton from Xenophyllum incisum, Biochem. Syst. Ecol., 35, 169-175, 2007.

Dwivedi, S., Khan, M., Srivastava, S. K., Syamasunnder, K. V., and Srivastava, A.: Essential oil composition of different accessions of Mentha x piperita L. grown on the northern plains of India, Flavour Frag. J., 19, 437-440, 2004.

Frizzo, C. D., Serafini, L. A., Dellacassa, E., Lorenzo, D., and Moyna, P.: Essential oil of Baccharis uncinella DC. from Southern Brazil, Flavour Frag. J., 16, 286-288, 2001.

Novak, J., Zitterl-Eglseer, K., Deans, S. G., and Franz, C. M.: Essential oils of different cultivars of Cannabis sativa L. and their antimicrobial activity, Flavour Frag. J., 16, 259-262, 2001.

Riu-Aumatell, M., Castellari, M., Lopez-Tamames, E., Galassi, S., and Buxaderas, S.: Characterisation of volatile compounds of fruit juices and nectars by HS/SPME and GUMS, Food Chem., 87, 627-637, 2004.

Tuberoso, C. I. G., Kowalczyk, A., Coroneo, V., Russo, M. T., Dessi, S., and Cabras, P.: Chemical composition and antioxidant, antimicrobial, and antifungal activities of the essential oil of Achillea ligustica All, J. Agric. Food Chem., 53, 10148-10153, 2005. 